\title{
MÉTHODOLOGIE : L'ENTRETIEN ETHNOGRAPHIQUE AU SERVICE DE L'ÉTUDE DES CIVILISATIONS POUR UNE ANALYSE COMPARÉE DES CULTURES FRANÇAISE ET SLOVAQUE
}

\author{
François Schmitt
}

\begin{abstract}
Ethnography can be useful to get a deeper knowledge of foreign civilizations. Ethnography enables the research worker to outgrow cultural stereotypes through the study of cultural representations. This is what is intended through a project of survey research: to ask questions to French and Slovak people about their views on the French culture and the Slovak culture. During this survey, the method of the non-directive interview will be used. The contribution is an introduction to the project and aims to present the methodology of the non-directive interview.

Keywords: culture; civilization; interview; ethnography; ethnology.

Résumé: Pour appronfondir les connaissances sur les civilisations étrangères, avoir recours à l'ethnographie permet de dépasser certains stéréotypes grâce à un travail sur les représentations du public étranger sur la culture cible. L'article est une introduction à un projet d'enquête sur la représentation des cultures française et slovaque par les Français et les Slovaques. Il traite de la méthode de l'entretien non directif, méthode bien adaptée à la future enquête. L'article présente les fondements conceptuels de la méthode de l'entretien non directif et la démarche de l'enquêteur au cours de l'entretien et après l'entretien.
\end{abstract}

Mots clés : culture ; civilisation ; entretien ; ethnographie ; ethnologie.

\section{Introduction}

Dans l'étude et l'enseignement des langues étrangères, la culture et la civilisation sont souvent abordées presqu'exclusivement à partir des sources écrites (articles de presse, extraits littéraires, études sociologiques ou historiques, manuels de civilisation, etc.) et audiovisuelles (extraits d'émissions de radio ou de télévision). Ces sources sont d'un apport précieux pour connaître une civilisation étrangère et permettent souvent de faire un tableau très complet de la culture cible. Si l'on s'en tient à ces sources, on risque pourtant d'avoir une vision stéréotypée voire fausse de la culture étudiée car on est tenté de faire des traits culturels immédiatemment visibles les caractéristiques majeures de la civilisation étudiée. Pour corriger cet effet déformant, il serait intéressant de soumettre le contenu des sources écrites et audiovisuelles au regard et aux représentations que l'étranger porte sur la culture cible. 
Dans cette perspective, l'ethnologie dispose d'outils et de méthodes pouvant rendre compte de la vision de l'autre portée sur une culture différente de la sienne. La méthode que nous jugeons particulièrement bien adaptée à ce type d'étude est l'entretien ethnographique et en particulier l'entretien non directif dont nous allons présenter dans cet article les démarches et les enjeux.

Notre projet de recherche est de réaliser une enquête sur la représentation des cultures française et slovaque par les Français et les Slovaques. Cet article constitue une introduction méthodologique à notre future enquête dont nous présenterons en conclusion les caractéristiques (terrain et échantillon). Son objectif est de montrer en quoi la méthode de l'entretien non directif est bien adaptée au thème de notre future enquête.

\section{Les origines de l'enquête ethnographique en ethnologie}

L'entretien non directif s'impose en sociologie comme méthode d'investigation à partir des années 1970 en opposition aux méthodes quantitatives classiques de recueil de données. Dans un même mouvement, on commence alors aussi à reconnaître chez les anthropologues et dans les années 1980 chez les sociologues, la subjectivité du chercheur. Paraissent depuis un certain nombre d'ouvrages théoriques mettant en avant la méthode non directive en l'opposant aux lacunes des méthodes dominantes quantitatives. Cette méthode reçoit diverses dénominations selon les auteurs : « entretien non directif » (Guy Michelat), «entretien ethnographique » (Stéphane Beaud), « entretien semi-directif » (Jean-Baptiste Legavre), « entretien compréhensif » (Jean-Claude Kaufmann), « entretien informel » (Patrick Bruneteau) (Memmi, 1999: 134).

La principale critique soulevée à l'encontre de cette méthode est sa trop grande part de subjectivité et la trop grande influence de l'enquêteur sur les réponses données par l'informateur. Les défenseurs de l'entretien non directif prennent le contre-pied de cette critique en mettant en avant la subjectivité même de la méthode qui, lorsqu'elle est prise en compte dans le travail de l'enquêteur, peut servir de fondement même à la méthode.

\section{Les fondements conceptuels de l'entretien non directif}

La méthode de l'entretien non directif nous semble bien adaptée à l'étude d'une civilisation étrangère et particulièrement à l'analyse comparée des cultures française et slovaque, non seulement parce qu'elle laisse une marge de manœuvre importante au chercheur, mais surtout parce qu' elle considère l'informateur comme la source essentielle de l'enquête. En étant centrée sur l'informateur, cette méthode de l'entretien non directif laisse donc une place importante aux représentations, aspect fondamental de notre enquête.

\section{1 «Un artisanat intellectuel»}

La standardisation du métier de sociologue, conséquence de l'avènement de la société de l'informatique, a tendance à se répercuter sur la pratique de l'entretien qui est mené de façon de plus en plus impersonnelle dans un souci d'objectivité. Jean-Claude Kaufmann s'oppose à cette évolution et veut, au contraire, faire de l'entretien non directif, qu'il appelle «entretien compréhensif », une méthode personnalisée (Kaufmann, 2007 : 13-14). Il revendique un « artisanat intellectuel », selon l' expression de W. Mills (1967), dans le métier de sociologue : le sociologue doit construire son œuvre et ne pas se laisser sou- 
Méthodologie : l'entretien ethnographique au service de l'étude des civilisations...

mettre par une méthodologie formaliste (Kaufmann, 2007 : 16). L'entretien non directif n'est donc pas une méthode formaliste mais laisse à l'enquêteur une certaine marge de manœuvre.

\subsection{L'effacement de l'enquêteur est illusoire}

La tendance est aujourd'hui à l'effacement de l'enquêteur pour ne pas influencer l'interviewé si bien que l'on s'achemine vers un entretien impersonnel standardisé : chaque entretien est conduit de la même manière. Il est finalement sans relief et son interprétation est alors réduite au minimum. La méthode de l'entretien standardisé travaille, en effet, sur les opinions de surface, immédiatement disponibles, elle n'atteint que les opinions flottantes sous des formes linguistiques appropriées alors qu' un entretien personnalisé est riche et complexe car il dissimule l'essentiel : les ratés de parole, les digressions, les dénégations, etc. Au contraire de l'entretien impersonnel, l'entretien non directif exige de l'enquêteur un engagement dans l'enquête, non seulement au moment de l'interprétation des données mais aussi pendant le déroulement de l'entretien (Kaufmann, 2007 : 19-20).

La nécessité de l'engagement de l'ethnographe dans son enquête devrait pousser l'enquêteur à réduire la distance le séparant du terrain. Cela ne signifie pourtant pas que la distanciation soit absente de l'enquête ethnographique : elle reste garante de l'objectivité de l'enquête. Mais elle n'intervient pas au stade de la collecte des données, étape de l'enquête où l'ethnographe est au contraire amené à entrer de plein pied dans le terrain. Elle se situe en amont, lorsque l'ethnographe découvre le terrain, et en aval, lors de l'analyse.

Lorsque l'enquêteur s'engage personnellement dans les entretiens, il doit accepter qu'une part de subjectivité interfère dans ses relations avec les informateurs et tenir compte de cela en repérant tout ce qui peut relever de la subjectivité de l'enquêteur durant les entretiens. Pour prendre en compte la subjectivité de l'enquêteur dans son travail, Michelat fait appel à la psychanalyse pour déceler les lapsus ou certaines formes de projection lors des entretiens (Michelat, 1975 : 241). Dans tous les cas de figure, qu'il y ait distance ou proximité entre l'ethnographe et l'informateur, il est donc indispensable de tenir compte des facteurs de subjectivité pouvant interférer sur l'entretien.

\subsection{L'informateur au cœur de l'enquête}

Tout en étant conscient de l'influence induite par sa présence dans l'enquête, l'enquêteur doit s'effacer le plus possible devant l'informateur qui doit jouer un rôle central dans l'enquête. En effet, partant du principe selon lequel la personne interrogée est la plus apte à explorer le champ du problème étudié, dans un entretien non directif l'enquêteur fait assumer à l'informateur l'exploitation du thème de la recherche alors que luimême n'a qu'un rôle de faciliteur ou de soutien. On considère donc l'informateur comme source du savoir car, comme tout un chacun, il est porteur d'une culture : chaque individu est porteur de la culture et des sous-cultures (en fonction des groupes sociaux qu'il fréquente, de sa personnalité, de son histoire, etc.) auxquelles il appartient. ${ }^{1}$ L'entretien $^{\prime}$ permet alors de révéler des modèles culturels intériorisés qui peuvent être appréhendés dans les productions verbales des informateurs. Si chaque individu est représentatif

Michelat reprend ici l'idée selon laquelle la réalité sociale est dans tout individu, thème développé par Norbert Elias, cf. Elias, Norbert (1991), La Société des individus, Paris : Fayard. 
de sa culture, les individus ne sont pourtant pas interchangeables car chacun perçoit la culture dont il est porteur à sa manière. L'enquêteur doit tenir compte de ce paradoxe : partir des comportements individuels pour étudier les comportements sociaux. En réalité, ce paradoxe n'est qu'apparant car on ne peut dissocier l'individuel du social. Selon Sapir « social ne s'oppose pas vraiment à individuel. Toute psychologie de l'individu est psychologie de la société, dans la mesure où le psychologique rend compte des conduites sociales $»^{2}$ (Michelat, 1975 : 232-234).

L'informateur ne révèle pourtant pas miraculeusement au cours de l'entretien une réalité sociale comme un livre ouvert mais construit le matériau ethnographique. C'est la dialectique de l'entretien qui participe à la construction de la réalité. En situation d'entretien, l'informateur est placé dans une situation exceptionnelle l'amenant à réfléchir sur sa propre vie. Pour rendre son discours cohérent, il met de l'odre dans ce qu'il révèle à l'enquêteur sur sa vie. Cette mise en ordre, souvent inconsciente, par l'informateur d'une réalité incohérente peut en apprendre beaucoup à l'enquêteur sur les contradictions qu'elle révèle chez l'informateur. Maître d'œuvre dans la construction du texte de l'entretien, l'informateur peut même glisser inconsciemment dans son discours des mensonges ou des vérités déformées sous forme de fables, ce dont l'enquêteur devra également tenir compte, en particulier au cours de l'analyse (Kaufmann, 2007 : 58-59).

\subsection{Les hypothèses de départ}

Le modèle classique de l'enquête consiste à élaborer a priori des hypothèses qui sont ensuite vérifiées sur le terrain pour être rectifiées par la suite. La méthode de l'entretien non directif inverse le processus : c'est à partir du terrain qu'on élabore les hypothèses. Deux attitudes sont cependant envisageables. Adopter la position extrême d'Anselm Strauss ${ }^{3}$ qui tire toutes ses hypothèses du terrain et s'immerge dans les faits sans formuler de question de départ. Jean-Claude Kaufmann, considérant au contraire qu'on risque ainsi de se noyer dans la richesse du terrain, préfère avoir une idée de départ en tête à la manière $\mathrm{d}^{\prime}$ Howard Becker qui propose une hypothèse 0 . Cette hypothèse 0 , même si elle peut être fausse, est un bon instrument de travail pour évoluer durant l'enquête car, pour Kaufmann, il faut quitter l'idée selon laquelle le terrain fait émerger de lui-même les idées (Kaufmann, $2007: 26)$.

\section{Mener un entretien non directif}

\subsection{Préparer l'entretien}

\subsubsection{Constituer l'échantillon}

Pour l'entretien standardisé quantitatif, l'échantillon est la pièce maîtresse et répond à des critères précis (âge, profession, situation familiale, résidence, etc.) car il est censé être représentatif de la population étudiée (Michelat, 1975 : 236). Pour l'entretien qualitatif non directif, par contre, l'échantillon peut être constitué de manière beaucoup plus souple. Comme nous l'avons montré plus haut, c'est l'individu qui est au cœur de l'enquête car il est représentatif de sa culture et parce que l'entretien s'appuie sur des histoires individuelles et non sur des statistiques chiffrées. L'échantillon peut donc être

\footnotetext{
2 Sapir, Eduard (1967), Anthropologie. Tome 1 : culture et personalité, Paris : Éditions de Minuit, 9.

3 Strauss, Anselm (1992), Grounded theory, Cambridge : University Press.
} 
Méthodologie : l'entretien ethnographique au service de l'étude des civilisations...

constitué d'un nombre assez réduit de personnes : 30 à 40 nous conseille Michelat. Mais l'échantillon ne peut pour autant être constitué n'importe comment: il faut qu'il soit pondéré et doit éviter les grands déséquilibres (Kaufmann, 2007 : 41-42). Les critères de sélection des informateurs - Michelat emploie à ce propos l'expression variables stratégiques - doivent être adaptés au thème de l'enquête au sens où les personnes interrogées doivent être choisies en fonction de leur représentativité (Michelat, 1975 : 236). Si l'échantillon est important, il n'est pourtant qu'un instrument de l'enquête dans laquelle l'analyse occupe autant de place.

\subsubsection{Préparer la grille des questions}

Dans l'entretien directif les questions sont imposées et l'enquêté n'a de choix qu'à y répondre. Or un questionnaire trop rigide risque de maintenir l'entretien à la surface, c'est-à-dire ne produire chez l'enquêté que des réponses superficielles et stéréotypées suggérées par les questions. Au contraire, laisser parler l'informateur librement - et les questions de l'enquêteur ne sont alors que des stimuli au discours de l'informateur permet d'atteindre le "niveau de profondeur des informations » (Michelat) qui se situe au niveau de la sphère socio-affective car, pour l'ethnographe, le niveau affectif révélé dans une conversation libre est au moins aussi important que ce qui est intellectualisé dans des réponses précises (Michelat, 1975 : 231). Il est pourtant conseillé de rédiger une vraie grille de questions avec de vraies questions classées par thèmes. Si le questionnaire doit être préparé de manière scrupuleuse et bien mémorisé par l'enquêteur, son utilisation au cours de l'entretien est cependant beaucoup plus souple. Les questions ne servent qu'à déclencher la conversation sur le thème désiré. Elles doivent certes se suivre logiquement pour ne pas déstabiliser l'informateur mais l'enquêteur peut aussi très bien modifier la grille au cours de l'entretien (Kaufmann, 2007 : 44-45).

\subsection{Le déroulement de l'entretien}

La méthode de l'entretien non directif, fondée sur des relations personnelles avec les informateurs, ne peut faire l'économie d'une réflexion sur les principes ethiques définis comme un ensemble de « règles ayant pour objet de soumettre l'activité scientifique au respect de valeurs jugées plus importantes que la liberté du chercheur » (Rossi). Pour garantir ces principes, l'enquêteur doit respecter certaines règles. Le principe fondamental de l'enquête est le respect de la confidentialité vis-à-vis de l'informateur qui peut être amené à livrer à l'enquêteur une part d'intimité (Dufoulon, 1999 : 14). L'enquêteur, qui est en situation de demandeur, laisse aussi à l'informateur le choix des armes : c'est à l'informateur de choisir le lieu et le moment de l'entretien et d'accepter ou non d'être enregistré. Enfin, l'enquêteur devrait jouer cartes sur table en exposant clairement à l'informateur l'objet et les pratiques de l'enquête, quitte à les discuter avec lui (Dufoulon, 1999 : 20).

Si l'informateur y consent, il est préférable d'enregistrer l'entretien car une simple prise de notes ne donne qu'un aperçu tronqué de la réalité dans la mesure où prendre des notes oblige l'enquêteur, qui ne peut tout noter, à procéder à une sélection immédiate en ne s'intéressant qu'aux informations de surface et en perdant ce qui constitue l'essentiel du message : les non-dits, les silences, les hésitations, les sous-entendus, etc. L'enregistrement peut rendre compte des différents niveaux du discours de l'informateur : il constituera le matériau de l'analyse du contenu, étape essentielle de l'enquête. 
Ces considérations éthiques nous rappellent à quel point l'enquêteur est tiraillé entre l'impératif prédateur de son métier qui est de tirer du terrain et de l'informateur un maximum d'informations et la dimension humaine et relationnelle de l'entretien qui n'est pas un interrogatoire mais tient davantage de la conversation entre égaux autour d'un thème. Dans cette perspective, l'enquêteur doit éviter deux écueils. Le premier serait d'énumérer ou lire les questions sur un ton monocorde car cela inciterait l'informateur à adopter le même style en donnant des réponses plates et brèves et le pousserait à se soumettre à l'enquêteur et à attendre passivement les questions. Le deuxième danger est de faire dériver la conversation vers un échange mou, une simple conversation. L'enquêteur doit donc rester maître du jeu : atteindre le ton de la conversation sans que l'entretien ne devienne réellement une conversation. Pour mener discrètement l'entretien, l'enquêteur doit faire preuve d'une grande capacité d'écoute de manière à pouvoir anticiper le déroulement de l'entretien en saisissant les points importants à développer dans le témoignage sans toutefois interrompre tout de suite l'informateur. Selon l'expression de Kaufmann, il faut mener "l'enquête dans l'enquête », c'est-à-dire trouver la meilleure question à poser en fonction de ce que dit l'informateur davantage qu'en fontion de la grille (Kaufmann, $2007: 47-49)$.

Un des objectifs de l'entretien étant de pénétrer dans le monde intime de l'informateur, l'enquêteur doit être capable de comprendre l'informateur, de pénétrer dans son système de penser en oubliant ses propres opinions et sa propre morale. Il doit être à l'écoute de l'informateur même s'il peut avoir l'impression que ce qu'il dit n'est pas intéressant. On appelle cette attitude, mêlant sympathie, compréhension (au sens moral du terme) et altruisme, l'empathie. Pour Kaufmann, l'enquêteur doit faire preuve d'empathie (Kaufmann, 2007 : 51). Pourtant, l'empathie comme qualité de l'enquêteur ne fait pas l'unanimité chez les ethnographes et les ethnologues. L'empathie est d'abord une notion ambiguë : pour Dufoulon, elle est «empreinte de culpabilité ethnocentrique » et met en question la relation d'égal à égal entre l'informateur et l'ethnographe (Dufoulon, 1999 : 22). Ensuite, la rencontre de l'ethnographe avec l'altérité est confrontation excluant toute empathie a priori : il n'y a pas « d'acculturation du chercheur » sur le terrain qui est plutôt un espace de traduction ou enquêteur et informateur vont rechercher un langage commun (Dufoulon, 1999 : 12-15).

Élément déclencheur de l'entretien, l'interaction entre l'ethnographe et l'informateur implique donc une relation dynamique entre enquêteur et enquêté excluant toute neutralité de l'enquêteur comme les tenants de la méthode classique le préconisent. La neutralité de l'enquêteur, "fantasme positiviste ", risque au contraire de mener l'entretien à l'échec. En effet, en s'effaçant, l'enquêteur empêche l'informateur, qui a besoin de repères, de se livrer suffisamment. Au contraire, en exprimant son opinion, l'enquêteur paraît aussi plus sincère aux yeux de l'informateur qui est alors incité à réagir (Kaufmann, $2007: 52$ ).

\section{Après l'entretien : l'analyse des contenus}

La partie essentielle de l'enquête est l'analyse des contenus, c'est-à-dire le décryptage des entretiens à partir des enregistrements audio ou, à défaut, des prises de notes.

Analyser un entretien ne signifie pas simplement en restituer le contenu à partir des enregistrements. C'est un travail d'investigation consistant à la mise à jour, à partir des entretiens, de concepts et d'hypothèses. L'analyse est donc une reconstruction de la réa- 
Méthodologie : l'entretien ethnographique au service de l'étude des civilisations...

lité (Kaufmann, 2007 : 72). Le matériau brut est constitué des enregistrements que certains ethnographes retranscrivent. C'est le cas de Michelat qui conseille aussi de relire plusieurs fois les retranscriptions pour s'en imprégner (Michelat, 1975 : 241). Kaufmann, quant à lui, pense que les retranscriptions orientent l'analyse sur l'écrit au détriment du paraverbal (rythme, intonation, silences, etc.) et préfère donc travailler directement à partir des enregistrements. Comme support écrit, intermédiaire entre le matériau brut (les enregistrements) et l'interprétation, Kaufmann rédige ensuite des fiches sur lesquelles il retranscrit les phrases les plus intéressantes et certains commentaires. Ces fiches ne constituent pourtant pas un matériau définitif : elles sont revues et sélectionnées à mesure que l'analyse progresse (Kaufmann, 2007 : 76).

L'analyse des contenus ne consiste pas simplement à lire les retranscriptions ou à écouter les enregistrements. Il s'agit d'interpréter le matériau, c'est-à-dire tirer du corpus un enseignement en partant du principe selon lequel tous les éléments du corpus, y compris les détails, ont leur importance (Michelat, 2007 : 237-238). Prendre en compte dans l'analyse tous les éléments du corpus permet de mieux repérer certaines phrases qui se répètent souvent d'un entretien à l'autre ou même au cours d'un même entretien : il s'agit de phrases socialement répandues auxquelles l'informateur a recours dans son argumentation de manière plus ou moins consciente et qui lui évitent d'invoquer un argument plus profond. Ces phrases passe-partout sont très intéressantes pour l'enquêteur car elles constituent des fragments de social peu personnalisés, sorte de " pot-pourri de notions disparates » (Kaufmann, $2007: 95)$.

Les éléments dégagés lors de l'interprétation n'acquièrent de signification que s'ils sont mis en relation entre eux. Cela renvoie à une conception de la culture inspirée du structuralisme selon laquelle chaque élément n'existe et ne peut être étudié que dans sa relation avec les autres éléments. C'est une analyse transversale du corpus qui permet de mettre les entretiens en relation entre eux. On procèdera alors à un couplage de lectures (ou écoutes) verticales (d'un seul entretien à la fois) et de lectures (ou écoutes) horizontales (de plusieurs entretiens à la fois de manière comparative). Le procédé n'est pas seulement technique mais répond à l'idée selon laquelle les entretiens se complètent les uns les autres car ils sont des interprétations différentes d'un même mythe. L'objectif de l'analyse transversale est ainsi de reconstituer le « raisonnement affectif » (non conscient) de l'informateur pour savoir quels rôles y jouent les modèles culturels. D'un point de vue méthodologique, cette lecture transversale d'un entretien à l'autre favorise aussi le questionnement de l'enquêteur (Michelat, 1975 : 238-243).

En poursuivant l'analyse du contenu plus en profondeur, l'enquêteur découvre que le caractère d'unité de l'informateur est trompeur. La déconstruction du récit de l'informateur pendant l'analyse révèle les contradictions et le caractère composite de l'informateur. Cette incohérence apparaît dans les contradictions contenues dans le discours de l'informateur. D'autres contradictions sont liées à la biographie de l'informateur qui, dans son discours, s'efforce de mettre de l'ordre dans son existence qui, elle non plus, n'est pas toujours cohérente (Kaufmann, 2007 : 97).

\section{Vers l'enquête de terrain : constituer l'échantillon}

Cette propédeutique méthodologique nous permettra de constituer l'échantillon en veillant à ce qu'il soit adapté au mieux à la méthode de l'entretien non directif et au terrain spécifique de notre future enquête. 
Nous réaliserons notre enquête en Slovaquie auprès d'un échantillon qui sera constitué de résidents français en Slovaquie et de Slovaques ayant des contacts réguliers avec la culture française. Les deux groupes d'informateurs français et slovaques seront sociologiquement équilibrés car ils seront tous les deux constitués de diplômés de l'enseignement supérieur exerçant des emplois qualifiés (enseignants, cadres). L'homogénéité des deux groupes ne pourra pourtant pas être assurée sur le point linguistique car la connaissance et la pratique de la langue de l'autre seront différentes d'un groupe à l'autre. Les Français résidant en Slovaquie n'ont généralement pas appris le slovaque avant de venir en Slovaquie et n'ont souvent qu'une connaissance imparfaite de la langue qu'ils ont apprise sur place. Il leur est même possible de séjourner en Slovaquie avec une maîtrise rudimentaire de la langue slovaque en ayant recours au français ou à d'autres langues véhiculaires, en général à l'anglais. Les Français de notre futur échantillon maîtrisent donc très inégalement le slovaque. Il est au contraire plus difficile de séjourner de manière prolongée en France sans bien parler français. Les Slovaques de notre futur échantillon maîtrisent donc généralement très bien le français. Nous pouvons ainsi d'emblée supposer que, si pour les Français, l'expérience de la culture slovaque ne s'accompagne pas forcément d'un apprentissage poussé de la langue slovaque, dans le cas des Slovaques, langue et culture françaises vont souvent de pair.

\title{
Bibliographie
}

Dufoulon, Serge (1999), "Marins et sociologues à bord du Georges Leygues, interactions de recherche ", Sociologie du travail 41, $\mathrm{n}^{\circ} 1,5-22$.

Kaufmann, Jean-Claude (2007), L'enquête et ses méthodes. L'entretein compréhensif, Paris : Armand Colin.

Memmi, Dominique - Arduin, Pascal (1999), «L'enquêteur enquêté. De la « connaissance par corps » dans l'entretien sociologique », Genèses 35, 131-145.

Michelat, Guy (1975), "Sur l'utilisation de l'entretien non directif en sociologie », Revue française de sociologie 16, 229-247.

Rossi, Ilario - Kaech, François - Foley, Rose-Anne - PAPadaniel, Yannis (2010) : L'éthique à l'épreuve d'une anthropologie en milieu paliatif : de l'insertion à la restitution, en ligne : www.ethnographiques.org, consulté le 21/11/2010.

\author{
François Schmitt \\ Katedra romanistiky \\ Fakulta humanitných vied \\ Univerzita Mateja Bela \\ Tajovského 40 \\ 97401 Banská Bystrica \\ Slovaquie \\ francois.schmitt@umb.sk
}

